\title{
Cervicogenic Dizziness Associated With Craniocervical Instability: A Case Report
}

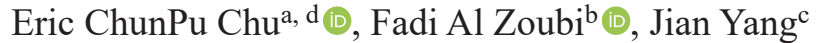

\begin{abstract}
Cervicogenic dizziness (CGD) is a clinical syndrome characterized by an illusory sensation of motion and disequilibrium secondary to neck pathologies. Reported here is the case of a 40 -year-old male presented with neck pain, dizziness, and aural symptoms in the past 12 months. The patient was previously diagnosed with Meniere's disease and treated with a low-sodium diet, betahistine and vestibular rehabilitation for 4 months, but the symptoms had not been relieved. Subsequently he sought out chiropractic evaluation. Radiographic clues illustrated a subtle anterolisthesis of the occiput on the cervical spine, manifested by a dissociation of the clivo-axial angle (CXA). After exclusion of other neurological, vascular and vestibular causes, craniocervical instability was considered as the cause of the CGD. He was treated with multi-component intervention consisting of spinal manipulation, intermittent motorized traction and ultrasound therapy to release cervical complaints. The patient reported salutary consequences to the 3-month treatment course with no neurologic sequelae. The current study serves as a vivid example of identifying CGD based on a subtle radiographic clue and by utilizing CXA as an assessment tool for craniocervical instability.
\end{abstract}

Keywords: Cervicogenic dizziness; Chiropractic; Clivo-axial angle; Craniocervical instability; Dizziness

\section{Introduction}

Cervicogenic dizziness (CGD) is a clinical syndrome characterized by an illusory sensation of motion and disequilibrium secondary to neck pathologies $[1,2]$. Although the etiology

Manuscript submitted September 15, 2021, accepted October 13, 2021

Published online November 5, 2021

${ }^{a}$ New York Chiropractic and Physiotherapy Centre, 41/F Langham Place Office Tower, 8 Argyle Street, Hong Kong, China

bepartment of Rehabilitation Sciences, The Hong Kong Polytechnic University, Hung Hom, Hong Kong, China

'LKS Faculty of Medicine, The University of Hong Kong, Hong Kong, China ${ }^{\mathrm{d} C o r r e s p o n d i n g ~ A u t h o r: ~ E r i c ~ C h u n P u ~ C h u, ~ N e w ~ Y o r k ~ C h i r o p r a c t i c ~ a n d ~ P h y s i-~}$ otherapy Centre, 41/F Langham Place Office Tower, 8 Argyle Street, Hong Kong, China. Email: eric@nymg.com.hk

doi: https://doi.org/10.14740/jmc3792 remains unknown, many cases of CGD have been associated with post whiplash injury, inflammatory, degenerative, or mechanical dysfunctions of the cervical spine [1]. It has been proposed that a disruption of the normal afferent signals or aberrant pain signals may affect the upper cervical proprioceptors to the vestibular nucleus resulting in disequilibrium in cases of CGD $[1,3]$. There is no standardized test for CGD. However, there are diagnostic criteria that may help to identify CGD: 1) The presence of neck pain before or at the same time of feeling dizzy $[2,4]$; 2) Successful management of cervical dysfunction leads to a simultaneous relief of dizziness [5]. The correlation between dizzy syndrome and neck movement is an explicit diagnostic criterion for CGD [2, 4]. Often, clinical features are blurred and diagnostic clues are subtle to be seen on imaging. An uncommon case of CGD associated with craniocervical instability $(\mathrm{CCI})$ is reported here. Excellent functional and radiographic outcome was achieved through non-operative measures.

\section{Case Report}

\section{Investigations}

A 40-year-old male radio presenter was evaluated for episodic spinning dizziness with aural fullness, tinnitus, and sudden hearing loss frequently affecting the right ear in the past 12 months. He also complained of chronic neck pain and occipital headache. He reported that episodes developed three times per week on average and caused spinning sensation of the room, lasting $2-3 \mathrm{~h}$. Episodes could sometimes be triggered by quick turning of the head, closing the eyes, or texting on a smartphone. The symptoms worsened in the evening and could be lessened by simply lying down. The patient was unable to host radio programs or carry out daily tasks as before, and this was why he sought medical help.

On previous neuro-otologic evaluation, tuning fork evaluation identified asymmetrical hearing loss. An audiogram confirmed a sensorineural hearing loss in the right ear. No pathologies of the auditory system were detectable. Blood tests, brain magnetic resonance imaging and neurological investigations did not show any abnormality. Based on the 2020 clinical practice guideline [6] (i.e., two vertiginous episodes with sensorineural hearing loss and fluctuating aural symptoms in the affected ear, after exclusion of other causes) our patient was diagnosed with Meniere's disease by his otolaryngolo- 

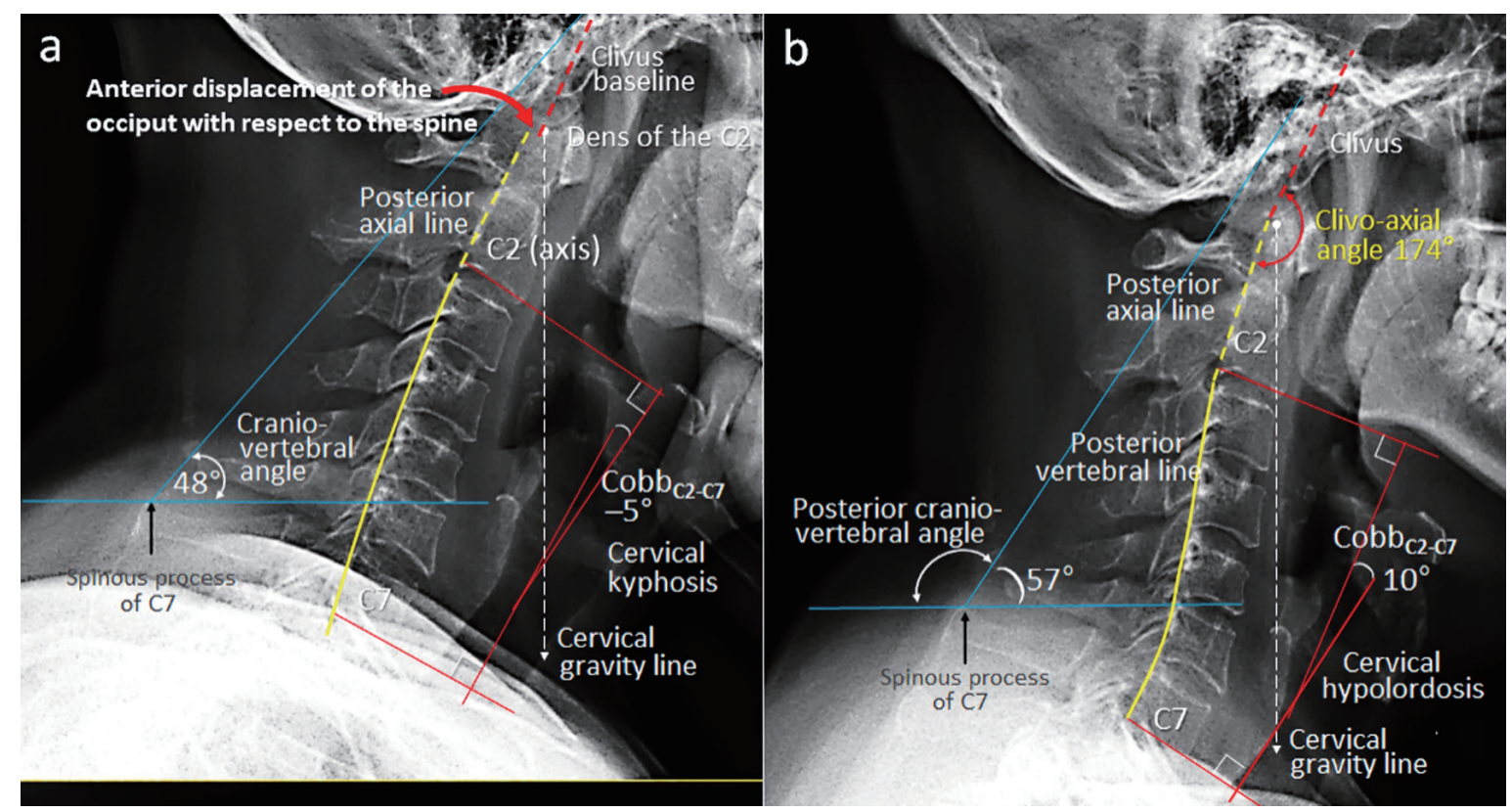

Figure 1. Comparison of cervical alignment on sagittal radiographs in neutral position. (a) At initial assessment, a lack of cervical lordosis, narrowing of the $\mathrm{C} 2 / 3, \mathrm{C} 5 / 6$ and $\mathrm{C} 7 / \mathrm{T} 1$ intervertebral spaces, and dissociation of the clivo-axial angle (CXA) were noticed (curved red arrow). The fulcrum effect at the tip of the dens might predispose the brainstem to impingement during neck motions. (b) At 12-month follow-up, all radiographic metrics showed significant improvement, including correction of the dissociated CXA, cervical curvature and other postural parameters (global lordosis angle, craniovertebral angle, head inclination and shifting of the cervical gravity line). Restored CXA and cervical alignment yielded simultaneous remission of neurological symptoms.

gist 4 months before he came to our clinic. Over the next 4 months the patient received oral betahistine to increase cochlear blood flow and reduce pressure in the endolymphatic space, diet modification, and vestibular rehabilitation provided by a physiotherapist. The severity of dizziness and the attacks frequency did not change. The patient presented for chiropractic assessment.

\section{Diagnosis}

On physical examination, the patient showed limited range of cervical motion $\left(40^{\circ} / 70^{\circ}\right.$ active extension and $60^{\circ} / 90^{\circ}$ of bilateral rotation). The self-reported peak pain intensity of his neck pain and headache was 7/10 on an 11-point numeric pain rating scale, where 0 means no pain and 10 means the worst imaginable pain [7]. The Dizziness Handicap Inventory (DHI) questionnaire was scored at 68/100 (mild, 0 - 30; moderate, 31 - 60; and severe, $61-100$ ) [8]. During the palpation, the patient reported tenderness at the $\mathrm{C} 2 / 3, \mathrm{C} 5 / 6, \mathrm{C} 7 / \mathrm{T} 1$ and $\mathrm{T} 5 / 6$ levels, and hypertonicity noted in bilateral upper trapezius, levator scapulae, supraspinatus, and pectoralis muscles. Other orthopedic, neurological, and neurodynamic tests were unremarkable. Cervical radiograph (Fig. 1a) showed a lack of cervical lordosis and narrowing of the $\mathrm{C} 2 / 3, \mathrm{C} 5 / 6$ and $\mathrm{C} 7 /$ T1 intervertebral spaces. The clivo-axial angle (CXA) [9], the angle between the posterior axial line and the clival line, was dissociated (curved red arrow in Figure 1a). The fulcrum effect at the tip of the dens might predispose the brainstem to impingement during neck motions. Ventral brainstem com- pression secondary to $\mathrm{CCI}$ was considered as the cause of CGD.

\section{Treatment}

The CXA varies from $150^{\circ}$ to $165^{\circ}$ in neutral position among adults [9]. An acute CXA of less than $150^{\circ}$ or being deformed like this case can be a source of ventral brainstem compression. Normalizing the CXA to reduce ventral brainstem compression is of paramount importance [10]. Although surgery is the direct procedure for removing neural compression, conservative strategy is the mainstay of initial care for patients with small amounts of CCI [11]. Conservative modalities for CCI include resting, pain relief, bracing, physiotherapy and spinal manipulation [12]. Non-surgical strategy for this case was based on a multicomponent approach which included: 1) Spinal manipulation to release the restricted cervical and thoracic levels; 2) Thermal ultrasound therapy to relieve muscle stiffness and restore motion; and 3) Cervical intermittent motorized traction to restore intervertebral spaces and decompress neural impaction. Treatment sessions were arranged three times a week for 3 months.

One month after starting treatment, the patient reported significant reduction of neck and head pains from $7 / 10$ to $2 / 10$ on an 11-point numeric pain rating scale. DHI score declined from $68 / 100$ to 20/100. Meanwhile, the patient reported that dizziness attacks had diminished from an average of three times to once weekly. In the next 2 months, he reported a reduction of the episodes of hearing lose, tinnitus, and perception 
of aural fullness gradually week by week. After the 3-month intensive treatment, the patient continued a maintenance treatment twice monthly for the next 9 months.

\section{Follow-up and outcomes}

At the 12-month follow-up, the patient was symptom free and his clinical metrics showed significant improvement. The patient reported neck pain reduced to $0 / 10$ on pain rating scale, the DHI score declined (0/100) and resolution of aural symptoms. The CXA had been restored (Fig. 1b). The cervical curvature and other postural parameters (global lordosis angle, craniovertebral angle, and shifting of the cervical gravity line) have been improved. These changes led to restoring the craniocervical stability which was positively reflected as well on other outcomes such as neck pain, cervical mobility and quality of life. The patient perceived the treatment with high satisfaction. No adverse events had occurred during the 12 months of care.

\section{Discussion}

The neck has a well-developed proprioceptive system [13]. These cervical proprioceptive inputs give significant somatosensory feedback maintaining postural balance [5]; and impairment of the cervical proprioception can cause a sensation of disequilibrium [2]. Various neck problems such as post whiplash injury, muscular fatigue, or cervical spondylosis can compromise this proprioceptive system and result in CGD [14]. Other postulated mechanisms responsible for producing the feeling of disequilibrium include compression of the vertebrobasilar artery, irritation of the cervical sympathetic nervous system, and aberrant pain signals from the upper cervical spine $[15,16]$.

CGD may cause dizzy symptoms similar to those of Meniere's disease. It has been assumed that CGD does not include aural symptoms like Meniere's disease [1]. However, recent studies demonstrated an association between cervical spine diseases and occurrence of aural dysfunctions manifesting as hearing loss, vertigo, and tinnitus $[17,18]$. A pilot study assessed aural symptoms among 42 neck pain patients showed a significant relationship between hearing loss and restricted range of cervical motion [17]. Another study of 60 participants suffering from cervical spondylosis further demonstrated a direct relation between the restricted side of cervical rotation and the affected side of hearing impairment [18]. Our patient had neck pain and a limitation of the cervical extension and bilateral rotation. Various mobility restrictions in the upper neck can lead to hearing impairment due to ear vessels and or nerves compromise [17].

CCI is a manifestation of ligamentous laxity between the skull and the first two cervical vertebrae, the atlas and the axis [19].The major ligaments involved are the alar, transverse and accessory ligaments. The line of reasoning that, anterolisthesis of the destabilized cranium on the cervical spine resulted in a backward displacement of the dense (the odontoid process of the axis), which might compress the brainstem or upper cervical cord, stretch the vertebral artery, and possibly alter venous or cerebrospinal fluid (CSF) outflow from the cranium [20]. Repetitive strain on the spinal cord/brainstem creates inflammatory edema which could cause irritation of the lower cranial nerves and dysfunction of the adjacent auditory organs [21].

Among the most frequently used landmarks to define the anatomic structure of the craniocervical junction are the clivus and axis [22]. The angle formed at the intersection of the clivus baseline with the posterior axial line is called the CXA $[9,20]$. Angular measurements relative to these structures are influenced by bony orientation, integrity of various ligaments, and paraspinal musculature [22]. The CXA is a useful index for healthcare providers to alert of the presence of CCI or brainstem compression [9]. A distorted CXA with the fulcrum effect at the tip of the dens can predispose the ventral brainstem to compression during neck motions (Fig. 1a). Recent studies have demonstrated the salutary consequences following surgical correction of the deformed CXA $[9,19]$.

Recent systematic reviews [23, 24] found a moderate clinical evidence to support the use of manual therapy to treat patients with CGD. In general, the studies reported significant reduction in dizziness intensity due to manual therapy as an intervention to treat CGD [24]. As seen in the current case, restoration of CXA and cervical alignment with simultaneous remission of clinical symptoms verified the diagnosis of CGD. It is possible that nonsurgical approaches could strengthen neck musculature, unload restricted structures, release neural irritation and restore spinal function [25]. However, it is often difficult to generalize results from a single case to a larger population. Further studies are required to corroborate this issue and to follow the patients' long-term outcomes.

\section{Conclusions}

The current study reported an association between CGD and CCI. The misalignment of craniocervical junction places stress on the brainstem and causes it to send confusing signals to the brain resulting in disequilibrium. The neurologic symptoms have been shown to correlate with CXA deformity and respond to the correction of clivo-axial misalignment.

\section{Acknowledgments}

None to declare.

\section{Financial Disclosure}

None to declare.

\section{Conflict of Interest}

None to declare. 


\section{Informed Consent}

Written informed consent was obtained from the patient for case publication.

\section{Author Contributions}

E. ChunPu Chu was mainly involved in diagnosis and treatment of this patient. F. Al Zoubiand and J. Yang drafted the report. All authors read and critically reviewed the manuscript, and then approved the final submitted version.

\section{Data Availability}

The authors declare that data supporting the findings of this study are available within the article.

\section{Abbreviations}

CCI: craniocervical instability; CGD: cervicogenic dizziness; CXA: clivo-axial angle

\section{References}

1. Reiley AS, Vickory FM, Funderburg SE, Cesario RA, Clendaniel RA. How to diagnose cervicogenic dizziness. Arch Physiother. 2017;7:12.

2. Chu ECP, Chin WL, Bhaumik A. Cervicogenic dizziness. Oxf Med Case Reports. 2019;2019(11):476-478.

3. Stanton TR, Leake HB, Chalmers KJ, Moseley GL. Evidence of impaired proprioception in chronic, idiopathic neck pain: systematic review and meta-analysis. Phys Ther. 2016;96(6):876-887.

4. Reid SA, Callister R, Katekar MG, Rivett DA. Effects of cervical spine manual therapy on range of motion, head repositioning, and balance in participants with cervicogenic dizziness: a randomized controlled trial. Arch Phys Med Rehabil. 2014;95(9):1603-1612.

5. Magnusson M, Malmstrom EM. The conundrum of cervicogenic dizziness. Handb Clin Neurol. 2016;137:365-369.

6. Basura GJ, Adams ME, Monfared A, Schwartz SR, Antonelli PJ, Burkard R, Bush ML, et al. Clinical practice guideline: Meniere's disease. Otolaryngol Head Neck Surg. 2020;162(2 suppl):S1-S55.

7. Jensen MP, McFarland CA. Increasing the reliability and validity of pain intensity measurement in chronic pain patients. Pain. 1993;55(2):195-203.

8. Grigol TA, Silva AM, Ferreira MM, Manso A, Gananca MM, Caovilla HH. Dizziness handicap inventory and visual vertigo analog scale in vestibular dysfunction. Int Arch Otorhinolaryngol. 2016;20(3):241-243.

9. Henderson FC, Sr., Henderson FC, Jr., Wilson WAt, Mark AS, Koby M. Utility of the clivo-axial angle in assessing brainstem deformity: pilot study and literature review.
Neurosurg Rev. 2018;41(1):149-163.

10. Henderson F, Sr., Rosenbaum R, Narayanan M, Mackall J, Koby M. Optimizing alignment parameters during craniocervical stabilization and fusion: a technical note. Cureus. 2020;12(3):e7160.

11. Chu EC, Wong AY, Lee LY. Craniocervical instability associated with rheumatoid arthritis: a case report and brief review. AME Case Rep. 2021;5:12.

12. Mathers KS, Schneider M, Timko M. Occult hypermobility of the craniocervical junction: a case report and review. J Orthop Sports Phys Ther. 2011;41(6):444-457.

13. Knapstad MK, Nordahl SHG, Goplen FK. Clinical characteristics in patients with cervicogenic dizziness: A systematic review. Health Sci Rep. 2019;2(9):e134.

14. Li Y, Peng B. Pathogenesis, diagnosis, and treatment of cervical vertigo. Pain Physician. 2015;18(4):E583-595.

15. Alqahtani MM, Kashoo FZ. Physical therapy in cervicogenic dizziness. Saudi J Health Sci. 2020;9(1):1-6.

16. Sung YH. Upper cervical spine dysfunction and dizziness. J Exerc Rehabil. 2020;16(5):385-391.

17. Vasaghi-Gharamaleki B, Naser Z. Predicting the risk of hearing impairment following the cervical spine diseases by measuring the cervical range of movements: a pilot study. Basic Clin Neurosci. 2017;8(5):413-418.

18. Karam BH, Hasan HS, Saeed HT. A study on the association of cervical spondylosis severity, as indicated by cervical motions, with hearing impairment. Biomed Hum Kinet. 2021;13(1):43-48.

19. Henderson FC, Sr., Francomano CA, Koby M, Tuchman K, Adcock J, Patel S. Cervical medullary syndrome secondary to craniocervical instability and ventral brainstem compression in hereditary hypermobility connective tissue disorders: 5-year follow-up after craniocervical reduction, fusion, and stabilization. Neurosurg Rev. 2019;42(4):915-936.

20. Henderson FC, Sr., Austin C, Benzel E, Bolognese P, Ellenbogen R, Francomano CA, Ireton C, et al. Neurological and spinal manifestations of the Ehlers-Danlos syndromes. Am J Med Genet C Semin Med Genet. 2017;175(1):195211.

21. Burcon MT. Upper cervical specific protocol and results for 139 patients with medically diagnosed Meniere's disease. J Vert Sublux Res. 2010;14:1-10.

22. Hussain I, Winston GM, Goldberg J, Curri C, Williams N, Chazen JL, Greenfield JP, et al. Impact of imaging modality, age, and gender on craniocervical junction angles in adults without structural pathology. J Craniovertebr Junction Spine. 2019;10(4):240-246.

23. Lystad RP, Bell G, Bonnevie-Svendsen M, Carter CV. Manual therapy with and without vestibular rehabilitation for cervicogenic dizziness: a systematic review. Chiropr Man Therap. 2011;19(1):21.

24. Yaseen K, Hendrick P, Ismail A, Felemban M, Alshehri MA. The effectiveness of manual therapy in treating cervicogenic dizziness: a systematic review. J Phys Ther Sci. 2018;30(1):96-102.

25. Chu EC, Lo FS, Bhaumik A. Secondary atlantoaxial subluxation in isolated cervical dystonia-a case report. AME Case Rep. 2020;4:9. 